\title{
Model-based Rendezvous Calibration of Mobile Sensor Networks for Monitoring Air Quality
}

\author{
Adrian Arfire, Ali Marjovi and Alcherio Martinoli \\ Distributed Intelligent Systems and Algorithms Laboratory \\ School of Architecture, Civil and Environmental Engineering \\ École Polytechnique Fédérale de Lausanne, Lausanne, Switzerland \\ Email: \{firstname.lastname\}@epfl.ch
}

\begin{abstract}
Mobile Wireless Sensor Networks (WSNs) hold the potential to constitute a real game changer for our understanding of urban air pollution, through a significant augmentation of spatial resolution in measurement. However, temporal drift, crosssensitivity and effects caused by varying environmental conditions (e.g., temperature) in low-cost chemical sensors (typically used in mobile WSNs) pose a tough challenge for reliable calibration. Based on state-of-the-art rendezvous calibration methods, we propose a novel model-based method for automatically estimating the baseline and gain characteristics of low-cost chemical sensors taking temporal drift and temperature dependencies of the sensors into account. The performance of our algorithm is evaluated using data gathered by our long-term mobile sensor network deployment, developed within the Nano-Tera.ch OpenSense II project $^{1}$ in Lausanne, Switzerland. We show that, in a realistic context of sparse and irregular rendezvous events, our method consistently improves rendezvous calibration performance for single-hop online calibration.
\end{abstract}

\section{INTRODUCTION}

The traditional method for monitoring air quality in urban settings is the use of high fidelity static stations located at selected sites. The high cost of the instrumentation used in these stations significantly limits their numbers, leading to very sparse monitoring networks. For example, the Swiss National Air Pollution Network (NABEL) uses a total of 16 stations for the whole country. This in turn limits the ability of the monitoring network to capture spatial heterogeneity of the pollution field which can be significant, particularly in urban settings.

WSNs employing low-cost sensing platforms have the potential of increasing the spatial resolution of measurement. Over the past decade there has been a growing interest in air quality monitoring using this type of platforms. This is reflected in a growing number of research projects aimed at high density air quality monitoring using static [1], [2], [3], or mobile WSNs [4], [5]. In this work we focus specifically on mobile networks which are particularly interesting as they permit covering a larger area with fewer sensor nodes.

Advances in sensor technology have led to a significant increase in the range of miniaturized, relatively cheap sensors commercially available for measuring various air quality parameters. However, the measurement quality of these sensors is typically much lower than that of traditional instrumentation. The most widely used chemical sensors for WSNs applications

\footnotetext{
${ }^{1}$ Nano-Tera.ch OpenSense II: http://opensense.epfl.ch
}

are either electrochemical or metal-oxide. Both of these classes of sensors suffer from temporal drift, different degrees of cross-sensitivity, and temperature, humidity or pressure dependence. Factory calibration is usually not provided, since the actual performance of the sensors will greatly depend on specific electronic integration and application domain, the latter determining the temperature, humidity and pressure in operational conditions.

The different options for performing the calibrations can be broadly classified as offline, or online methods. The first class includes one-off initial calibration methods, by experimental evaluation with a parallel reference instrument before deployment [6], [7]. The success of this type of methods depends on the ability of the experimental set-up to re-create the variability of all the relevant influencing parameters. While this might be feasible, although not trivial, for characterizing cross-sensitivities and the effect of environmental parameters, accurately capturing temporal drift behavior can imply impractically long experiments. An option for dealing with this issue would be replacing the one-off calibration scheme with a periodic offline calibration, but when considering large scale deployments this would be too time-consuming to be practical.

Online calibration algorithms are automatic methods for estimating the sensor calibration parameters during deployment. To state the problem, consider a set of low-cost mobile sensor nodes each having its own specific characteristics in terms of temporal drift, cross sensitivity to other modalities, and environmental parameter dependencies. The question is how to calibrate them in order to have consistently accurate measurements. Mobile WSNs provide an opportunity for performing this type of calibration by leveraging spatiotemporal encounters between pairs of sensor nodes, called rendezvous, and using the measurements gathered within these events to improve the calibration parameter estimate. If no reference signal is available this type of methods can provide a relative calibration of the network (i.e., ensuring consistency between sensor nodes). If rendezvous with a reference station are available, these methods can also provide an absolute calibration. In this paper, we assume that there is at least one reference sensing station which the mobile sensors encounter from time to time. Using the measurements taken by the sensors and the reference station, a statistical regression method can potentially provide a calibration function. In this paper, we study how to integrate the characteristics of the sensors in terms of time-drift and temperature dependencies into models to be used in the 
calibration process and what is the subsequent impact on the calibration performance.

Pioneering contributions to the field of online rendezvous calibration are the works of Hasenfratz et al [8] and Saukh et al [9], [10]. In [8], the authors introduce and compare three types of online algorithms (forward, backward, and instant calibration) based on least squares temporal-weighted regression of calibration tuples (i.e. pairs of reference and raw sensor measurements). They evaluate the performance of these algorithms by considering both simulated and real measurements, with uniformly distributed simulated rendezvous events. In [9], Saukh et al give a rigorous definition for rendezvous events and introduce the concept of rendezvous connection graphs, studying their properties in the context of sensor fault detection and sensor calibration. More recently, in [10], the authors address the problem of error accumulation in multi-hop calibration algorithms that use ordinary least squares regression, and propose the use of geometric mean regression as a better alternative to the former method.

Both [9] and [10] consider a linear relationship between sensor readings and the targeted concentration level, using only two parameters: the sensor's baseline and its gain. Based on this assumption, the proposed algorithms attempt to estimate these parameters directly, and assume them to be close to constant between calibration cycles. The sensor model considered in [8] is a generalized polynomial relating sensor readings to gas concentrations. The effect of environmental parameters or the temporal drift are not included in the model, although the latter is taken into account by the weighing mechanism of the proposed calibration algorithms.

We propose a different approach to the calibration problem by placing a direct focus on the sensor model selection and its impact on calibration performance. In particular, we consider models of varying complexity for electrochemical sensors and systematically evaluate them, based on long-term measurement series recorded within the Nano-Tera.ch OpenSense II project. We propose a sliding-window model-based rendezvous calibration algorithm and evaluate its performance for different sensor models and different assumptions on the calibration window size. Using realistically emulated rendezvous, we show that, in the context of sparse rendezvous events and assuming relatively predictable sensor behavior, there is a significant performance benefit in the use of more complex sensor models.

\section{THE SENSOR MODEL}

The general form for a model describing the behavior of a chemical sensor can be expressed as:

$$
s(t)=f(t, y(t), \mathbf{x}(t), \mathbf{w}(t), e(t))
$$

where:

- $\quad t$ represents the time variable

- $s(t)$ is the sensor reading

- $y(t)$ is the true concentration value of the targeted chemical

- $\quad \mathbf{x}(t)$ is the vector of chemical signals to which the sensor is cross-sensitive

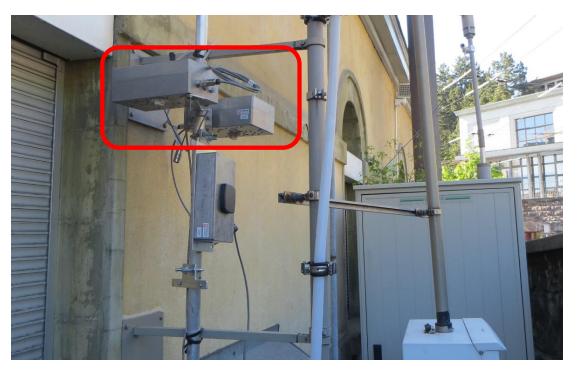

Fig. 1. Two OpenSense II sensor nodes deployed in parallel besides Lausanne NABEL station.

- $\quad \mathbf{w}(t)$ is the vector of environmental parameters (e.g., temperature, relative humidity, etc.) which have an effect on the sensor

- $\quad e(t)$ is a zero-mean independent noise.

As most chemical sensors are designed to have a linear dependence on $y(t)$, we introduce the baseline and gain functions $b(\cdot), g(\cdot)$, and re-write Eq. 1 as:

$$
s(t)=b(t, \mathbf{w}(t))+g(t, \mathbf{w}(t)) \cdot y(t)+f_{x}(t, \mathbf{x}(t), \mathbf{w}(t))+e(t)
$$

Furthermore, if the sensor has a good selectivity for the targeted chemical, then $f_{x}(\cdot)$ can be dropped, leading to:

$$
s(t)=b(t, \mathbf{w}(t))+g(t, \mathbf{w}(t)) \cdot y(t)+e(t)
$$

In this case, calibrating the sensor means obtaining the estimates $\bar{b}(\cdot), \bar{g}(\cdot)$ of the sensor baseline and gain, in order to infer the targeted chemical concentration:

$$
\bar{y}(t)=(s(t)-\bar{b}(t, \mathbf{w}(t))) / \bar{g}(t, \mathbf{w}(t))
$$

In previous work on rendezvous calibration, the gain and bias functions were considered to be constant between calibration cycles [8], [10]. The significance of this assumption on calibration performance depends on the inter-play between the degree of sensitivity to environmental factors, drift dynamics, and the frequency of rendezvous events with a reference station. To investigate this, we focused on studying one of the sensors used in the OpenSense II project, namely the City Technology A3CO carbon monoxide sensor [11].

The A3CO is a highly selective 4-electrode electrochemical sensor, which means that the general model structure in Eq. 3 can be assumed. As is the case with other electrochemical sensors, temperature variations can affect both the sensor gain and its baseline. Despite the fact that the sensor's forth electrode is meant to compensate for temperature effects on the baseline, its use does not completely eliminate temperature influence. The sensor is also sensitive to pressure variations. While this might be a problem when using pump-driven sampling, for the passive sampling system considered in this work, this was not an issue. Finally, the sensor manufacturer states that there is no significant effect of humidity variation on the sensor performance, which was confirmed by our experiments.

To derive a list of the candidate sensor models we have used data from sensor nodes deployed for long periods at the NABEL station in Lausanne (see Fig. 1), whose time series are used for ground truth purpose. These deployments were done in multiple 


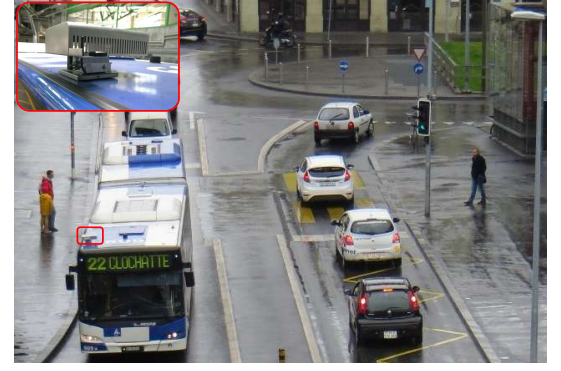

Fig. 2. Our sensor node anchored to a Lausanne bus.

stages, the first prototype station being installed in June, 2010. Apart from the $\mathrm{A} 3 \mathrm{CO}$ readings and the NABEL reference for $\mathrm{CO}$, we also record with our node the temperature and relative humidity. Based on the sensor specifications and our analysis of the collected time series, we propose the following candidate sensor models of increasing complexity, which include explicit consideration of temporal drift and dependence on temperature (denoted as $T(t)$ ):

$$
\begin{gathered}
m_{1}: s(t)=p_{0}+p_{1} \cdot y(t) \\
m_{2}: s(t)=p_{0}+p_{1} \cdot y(t)+p_{2} \cdot t \\
m_{3}: s(t)=p_{0}+p_{1} \cdot y(t)+p_{2} \cdot t+p_{3} \cdot T(t) \\
m_{4}: s(t)=p_{0}+\left(p_{1}+p_{2} \cdot T(t)\right) \cdot y(t)+p_{3} \cdot t+p_{4} \cdot T(t) \\
m_{5}: s(t)=p_{0}+p_{1} \cdot y(t)+p_{2} /\left(p_{3}+t\right)+p_{4} \cdot T(t) \\
m_{6}: s(t)=p_{0}+\left(p_{1}+p_{2} \cdot T(t)\right) \cdot y(t)+p_{3} /\left(p_{4}+t\right)+p_{5} \cdot T(t)
\end{gathered}
$$

\section{MODEL-BASED RENDEZVOUS CALIBRATION}

In this section, we propose a model-based rendezvous calibration algorithm. Its working principle is simple and similar to the forward calibration algorithm described in [8], as it also uses rendezvous data tuples gathered within a certain time window to obtain the calibration parameters at a given time. The main distinctive features comes from the fact that instead of weighing measurement tuples based on their age and regressing directly for the calibration parameters, our algorithm first estimates the sensor model parameters.

We use a more restrictive definition for a rendezvous than the one presented in [9]: two stations are considered to have had a rendezvous event only if they are present at the same time on the same street link (i.e. on the same segment of a street, between two junctions). The justification for opting for street-link instead of disk-based rendezvous can be found in [12], where we discuss the fact that in an urban environment measurement correlations are based on traffic flows through the street network more than on the geographic distance. In this work, for simplicity, we only consider single-hop calibration, i.e. calibration exclusively with the reference NABEL station.

\section{EVALUATION}

To evaluate the performance of the proposed calibration method and its sensitivity to sensor model choice and calibration window size, we devise the following set-up: we use one of the static sensor nodes deployed at the NABEL station as a mock mobile station and we simulate rendezvous events based on realistic probability functions. The advantage of this approach
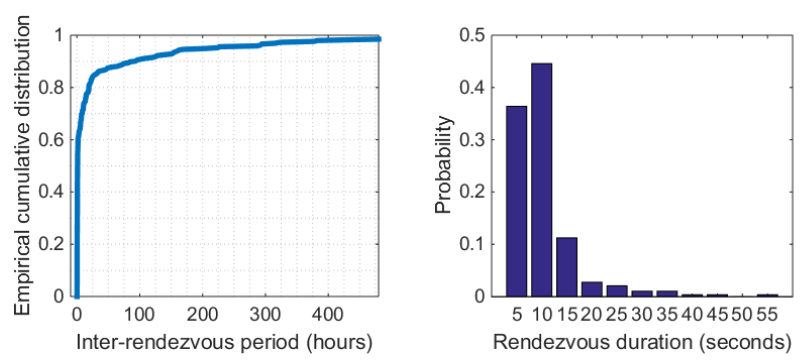

Fig. 3. Empirical cumulative distribution function for inter-rendezvous periods (left) and probability mass function of rendezvous duration (right) are derived from the OpenSense II mobile sensor network

is that the ground truth is known throughout the experiment, allowing us to calculate the performance of the algorithm also between calibration cycles. Furthermore, this set-up allows us to isolate the performance evaluation of the algorithm from currently not modeled effects of platform mobility.

Unlike the scenario described in [8], the NABEL station in Lausanne is not located on a regular bus route, and the rendezvous with it are therefore sparse and irregular. To derive realistic probability functions for sampling our simulated events, we have used the mobility data generated by the OpenSense II bus nodes (see Fig. 2) since their deployment, approximately 22 months ago. Considering only rendezvous events between one bus and the reference station, we calculated the empirical cumulative distributed function of the inter-rendezvous period and also the probability mass function of the length, or duration, of one rendezvous (see Fig. 3). Based on these functions we simulate realistic rendezvous sequences for our deployment.

Before applying the calibration algorithm, we first tested the quality of each of the models presented in Section II, by fitting them through least squares regression on the time series gathered from our nodes deployed near the NABEL station. We evaluated their performance through a 10 -fold cross-validation technique. The model fitting was done both on the full time range, but also on decreasing temporal sections (i.e. monthly, weekly, daily). The performance metric used was the Root Mean Squared Error (RMSE) of the calibrated series. An exemplar result is presented in Fig. 4 for the sensor which was the longest continuously deployed at NABEL (approximately 10 months).

For the complete time range, the more complex models outperformed the simpler ones, although adding a temperature effect to the gain in models $m_{4}$ and $m_{6}$ did not produce a significant improvement, and they were subsequently discarded. As the temporal calibration window is reduced, the benefit of the more complex models is gradually lost, and while for the monthly and weekly scales having at least an explicit temporal component still brings a marginal benefit, this disappears completely at the daily scale. This performance trend is justifiable by the fact that, at shorter time scales, the temperature excursion is also limited, making it more difficult to accurately estimate its impact. Looking at the temporal drift, an interesting aspect is the fact that, at the monthly scale, the hyperbolic models perform worse than the linear ones. This is most probably due to the fact that a hyperbola is harder to estimate than a line when reducing the number of regression samples.

We ran the calibration algorithm, varying the calibration 


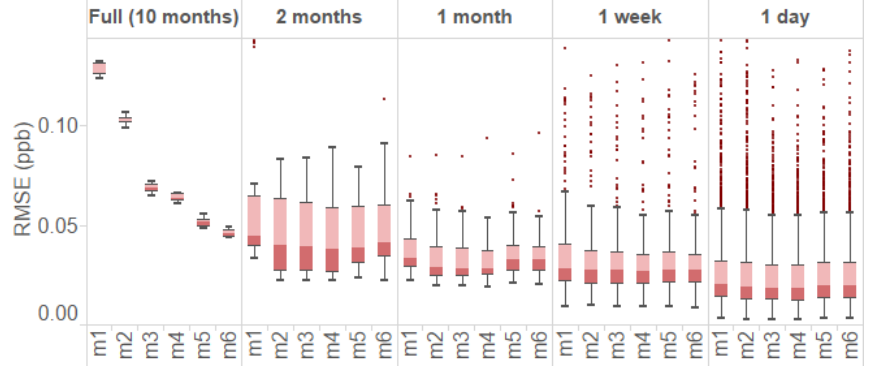

Fig. 4. Results of fitting the 6 sensor models on different time scales, using sensor data gathered over 10 months near the reference NABEL station. 90\% of all available data is used for fitting the models, and $10 \%$ for validation.

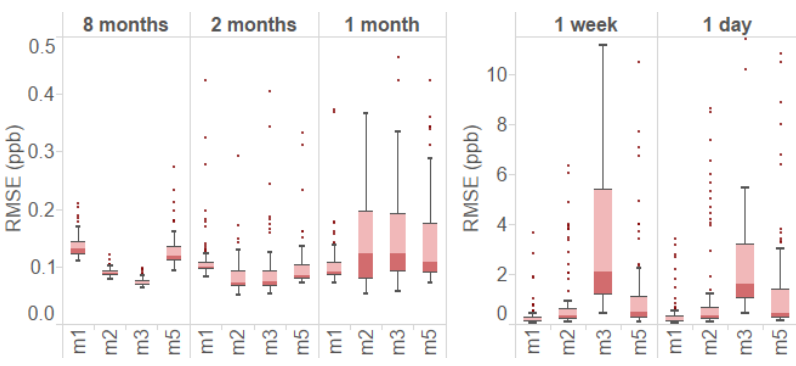

Fig. 5. Evaluation of model-based rendezvous calibration algorithm for the four selected models using calibration windows of different sizes. The performance drops, particularly for short calibration windows because of the rendezvous distribution.

window from one day to 8 months and using all models except $m_{4}$ and $m_{6}$. For each combination of the set-up parameters we performed 100 randomized runs. The results of these experiments are shown in Fig. 5. Due to the scarcity of data, for the short calibration windows the complex algorithms perform poorly, but as the window increases the situation is reversed. In fact, the performance obtained by complex algorithms with wide calibration windows is superior to the one obtained by the simplest model on short windows. The main drawback of using the complex models is the necessary size of the window in itself, which implies that the system needs to wait for a long time before being able to do the first calibration. One practical solution to this problem is of course, to use both types of models: the less complex ones during the early phase of the deployment and the more complex one as it matures.

These results are, of course, relevant for a scenario of sparse rendezvous events and the performance of the algorithm will tend to the regression results presented in Fig. 4, as rendezvous become more frequent.

\section{CONCLUSION}

In this paper, we proposed a model-based approach to online rendezvous calibration of mobile air quality monitoring networks. We started by studying the long-term performance of the electrochemical sensors used in the OpenSense II project for monitoring carbon monoxide, and derived models with increasing levels of complexity. These included models that explicitly take into account temporal drift and temperature effects. We then evaluated the ability of each of these models to approximate the sensor behavior at different temporal scales and found that the more complex models are more accurate when considering relatively large temporal scales. As the temporal scale is reduced, the benefit of the more complex models is gradually lost, and while for the monthly and weekly scales having at least an explicit temporal component still brings a marginal benefit, this disappears completely at the daily scale.

We subsequently proposed and implemented a slidingwindow single-hop rendezvous calibration algorithm based on the selected models. We evaluated its performance for different models and varying calibration window sizes, with a set-up that used realistically simulated rendezvous sequences and real measurement data from a static station. Our results suggest that the complexity of the model should be carefully chosen as a function of the temporal distribution of calibration opportunities. While in this work our approach was heuristic, we are interested in the further development of statistical methods that would be able to tune the model complexity in order to maximize calibration performance.

\section{ACKNOWLEDGMENT}

This work was funded by NanoTera.ch, a research initiative scientifically evaluated by the Swiss National Science Foundation and financed by the Swiss Confederation, in the framework of the OpenSense II project.

\section{REFERENCES}

[1] Centre for Scientific Computing, University of Cambridge. Sensor Networks for Air Quality at Heathrow Airport. [Online]. Available: http://www.snaq.org/

[2] R. N. Murty, G. Mainland, I. Rose, A. R. Chowdhury, A. Gosain, J. Bers, and M. Welsh, "Citysense: An urban-scale wireless sensor network and testbed," in IEEE Conference on Technologies for Homeland Security, 2008, pp. 583-588.

[3] L. Sánchez, V. Gutiérrez, J. A. Galache, P. Sotres, J. R. Santana, J. Casanueva, and L. Muñoz, "Smartsantander: Experimentation and service provision in the smart city," in 16th International Symposium on Wireless Personal Multimedia Communications, 2013, pp. 1-6.

[4] K. Aberer, S. Sathe, D. Chakraborty, A. Martinoli, G. Barrenetxea, B. Faltings, and L. Thiele, "Opensense: open community driven sensing of environment," in ACM SIGSPATIAL International Workshop on GeoStreaming, 2010, pp. 39-42.

[5] N. Castell, H.-Y. Liu, M. Kobernus, A. J. Berre, J. Noll, E. Cagatay, and R. Gangdal, "Mobile technologies and personalized environmental information for supporting sustainable mobility in oslo: The citi-sensemob approach," in 28th EnviroInfo Conf., Germany, 2014.

[6] M. C. Carotta, G. Martinelli, L. Crema, C. Malagù, M. Merli, G. Ghiotti, and E. Traversa, "Nanostructured thick-film gas sensors for atmospheric pollutant monitoring: quantitative analysis on field tests," Sensors and Actuators B: Chemical, vol. 76, no. 1, pp. 336-342, 2001.

[7] M. Kamionka, P. Breuil, and C. Pijolat, "Calibration of a multivariate gas sensing device for atmospheric pollution measurement," Sensors and Actuators B: Chemical, vol. 118, no. 1, pp. 323-327, 2006.

[8] D. Hasenfratz, O. Saukh, and L. Thiele, "On-the-fly calibration of low-cost gas sensors," Wireless Sensor Networks, pp. 228-244, 2012.

[9] O. Saukh, D. Hasenfratz, C. Walser, and L. Thiele, "On rendezvous in mobile sensing networks," in Real-World Wireless Sensor Networks, 2014, pp. 29-42.

[10] O. Saukh, D. Hasenfratz, and L. Thiele, "Reducing multi-hop calibration errors in large-scale mobile sensor networks," in 14th International Conference on Information Processing in Sensor Networks, Seattle, WA, USA, Apr 2015.

[11] City Technology Ltd. A3CO Sensor Datasheet. [Online]. Available: https://www.citytech.com/PDF-Datasheets/a3co.pdf

[12] A. Marjovi, A. Arfire, and A. Martinoli, "High resolution air pollution maps in urban environments using mobile sensor networks," in 11th International Conference on Distributed Computing in Sensor Systems, 2015, pp. 11-20. 